\title{
PREDICTING THE DIRECTION OF THE FINAL SPIN FROM THE COALESCENCE OF TWO BLACK HOLES
}

\author{
EnRico Barausse ${ }^{1}$ and Luciano Rezzolla ${ }^{2,3}$ \\ ${ }^{1}$ Center for Fundamental Physics, University of Maryland, College Park, MD 20742-4111, USA \\ ${ }^{2}$ Max-Planck-Institut für Gravitationsphysik, Albert-Einstein-Institut, Potsdam-Golm, Germany \\ ${ }^{3}$ Department of Physics and Astronomy, Louisiana State University, Baton Rouge, LA, USA \\ Received 2009 April 15; accepted 2009 August 31; published 2009 September 25
}

\begin{abstract}
Knowledge of the spin of the black hole resulting from the merger of a generic black-hole binary is of great importance for studying the cosmological evolution of supermassive black holes. Several attempts have been made to model the spin via simple expressions exploiting the results of numerical-relativity simulations. While these expressions are in reasonable agreement with the simulations, they neglect the precession of the binary's orbital plane, and cannot therefore be applied directly-i.e., without evolving the system to small separations using post-Newtonian theory-to binaries with separations larger than a few hundred gravitational radii. While not a problem in principle, this may be impractical if the formulas are employed in cosmological merger trees or $N$-body simulations, which provide the spins and angular momentum of the two black holes when their separation is of hundreds or thousands of gravitational radii. The formula that we propose is instead built on improved assumptions and gives, for any separation, a very accurate prediction both for the norm of the final spin and for its direction. By comparing with the numerical data, we also show that the final-spin direction is very accurately aligned with the binary's total angular momentum at large separation. Hence, observations of the final-spin direction (e.g., via a jet) can provide information on the binary's orbital plane at large separations and could be relevant, for instance, for studying X-shaped radio sources.
\end{abstract}

Key words: black hole physics - galaxies: nuclei - gravitational waves - relativity

\section{INTRODUCTION}

While analytic solutions for isolated black holes $(\mathrm{BHs})$ have a long history, the dynamics of $\mathrm{BH}$ binaries has been solved only recently and through computationally expensive numerical-relativity (NR) calculations (see Pretorius 2007 for a review). Despite the mathematical complexity of the problem, many results of the NR simulations can be reproduced accurately using semianalytical prescriptions (Damour \& Nagar 2009; Buonanno et al. 2009) based on post-Newtonian (PN) and $\mathrm{BH}$ perturbation theory. It is therefore not entirely surprising that the dimensionless spin of the remnant from a BH-binary merger, $\boldsymbol{a}_{\mathrm{fin}}=S_{\mathrm{fin}} / M_{\mathrm{fin}}^{2}$, can be described, with increasing accuracy, via simple prescriptions based on point particles (Hughes \& Blandford 2003; Buonanno et al. 2008; Kesden 2008), on fits to the NR data (Rezzolla et al. 2008b, 2008c; Tichy \& Marronetti 2008; Boyle et al. 2008; Boyle $\&$ Kesden 2008), or on a combination of the two approaches (Rezzolla et al. 2008a; see Rezzolla 2009 for a review). These formulas are useful because they provide information over the entire seven-dimensional parameter space for $\mathrm{BH}$ binaries in quasi-circular orbits, namely, the mass ratio $q \equiv M_{2} / M_{1}$ and the six components of the initial dimensionless spin vectors $\boldsymbol{a}_{1,2}=\boldsymbol{S}_{1,2} / M_{1,2}^{2}$. Such parameter space could in principle be investigated entirely via NR calculations; in practice, however, the simulations are still very expensive and restricted to $q=0.1-1$. Also, these formulas have many applications: in astrophysics, where they could provide information on massive-star binaries (Miller et al. 2009); in cosmology, where supermassive BHs (SMBHs) are believed to assemble through accretion and mergers (Berti \& Volonteri 2008); in gravitational-wave astronomy, where a priori knowledge of the final spin can help the detection (Berti et al. 2007a).

While the different expressions for the spin norm, $\left|\boldsymbol{a}_{\text {fin }}\right|$, are in good agreement among themselves and with the numerical data, the predictions for the final-spin direction, $\hat{\boldsymbol{a}}_{\mathrm{fin}} \equiv \boldsymbol{a}_{\mathrm{fin}} /\left|\boldsymbol{a}_{\mathrm{fin}}\right|$, do not agree well with one another. Moreover, all expressions are built from and model the typical "NR binaries" and hence the dynamics of the last few orbits before the merger, and do not account systematically for the precession of the orbital angular momentum $\boldsymbol{L}$, thus becoming imprecise when the binary is widely separated (Tichy \& Marronetti 2008). Of course, it is possible to use the PN equations and evolve a widely separated binary to small separations, read off the relevant information and apply the presently available formulas (this was suggested by Tichy \& Marronetti 2008, who correctly remark that their expression is valid only at small separations). While not a problem in principle, this procedure can be impractical in applications, such as cosmological merger trees or $N$-body simulations, that provide the spins of the two BHs at separations of hundreds or thousands of gravitational radii.

We follow here a different approach, and using assumptions slightly different from those made in Rezzolla et al. (2008a), we present a new expression for $\boldsymbol{a}_{\text {fin }}$ which is applicable to binaries with arbitrary separations and which also provides better results for the final-spin direction at small separations.

\section{DERIVATION OF THE FORMULA}

We recall that when the BHs have spins that are aligned with $\boldsymbol{L}$, the NR results are accurately described by (Rezzolla et al. 2008a)

$$
a_{\mathrm{fin}}=\tilde{a}+\tilde{a} v\left(s_{4} \tilde{a}+s_{5} v+t_{0}\right)+v\left(2 \sqrt{3}+t_{2} v+t_{3} v^{2}\right)
$$

where $v \equiv M_{1} M_{2} /\left(M_{1}+M_{2}\right)^{2}$ is the symmetric mass ratio and $\tilde{a} \equiv\left(a_{1}+a_{2} q^{2}\right) /\left(1+q^{2}\right)$. The five coefficients $t_{0}, t_{2}, t_{3}, s_{4}$, and $s_{5}$ in Equation (1) can be determined straightforwardly by fitting the results of the NR calculations. However, an additional condition can be employed by using the results obtained by Scheel et al. (2009) for equal-mass non-spinning BHs and thus enforce that for $a_{1}=a_{2}=0, v=1 / 4$ and to the claimed 
precision

$$
a_{\mathrm{fin}}=\frac{\sqrt{3}}{2}+\frac{t_{2}}{16}+\frac{t_{3}}{64}=0.68646 \pm 0.00004 .
$$

This leaves only four unconstrained coefficients, so that by using the NR results for the 72 aligned binaries published so far (Rezzolla et al. 2008b, 2008c; Buonanno et al. 2007; Berti et al. 2007b, 2008; Gonzalez et al. 2009; Scheel et al. 2009) we obtain

$$
\begin{aligned}
& s_{4}=-0.1229 \pm 0.0075, \quad s_{5}=0.4537 \pm 0.1463, \\
& t_{0}=-2.8904 \pm 0.0359, \quad t_{3}=2.5763 \pm 0.4833,
\end{aligned}
$$

with an agreement $\left|a_{\text {fin }}^{\mathrm{NR}}-a_{\text {fin }}^{\text {fit }}\right| \leqslant 0.0085$ with the data ${ }^{4}$; using the constraint (2) we then also obtain $t_{2}=-3.5171 \pm 0.1208$. Because of the larger data set used, the values in Equation (3) are slightly different from those in Rezzolla et al. (2008a).

Because Equation (1) provides information over only three of the seven dimensions of the parameter space, we will next show how to cover the remaining four dimensions and thus derive an expression for $\boldsymbol{a}_{\mathrm{fin}}$ for generic $\mathrm{BH}$ binaries in quasi-circular orbits. Following the spirit of Rezzolla et al. (2008a), we make the following assumptions.

I. The mass $M_{\mathrm{rad}}$ radiated to gravitational waves can be neglected, i.e., $M_{\text {fin }}=M \equiv M_{1}+M_{2}$. The radiated mass could be accounted for by using the NR data for $M_{\text {fin }}$ (Tichy \& Marronetti 2008) or extrapolating the test-particle behavior (Kesden 2008). The reason why assumption (I) is reasonable is that $M_{\mathrm{rad}}$ is largest for aligned binaries, but these are also the ones employed to fit the free coefficients in Equation (3). Therefore, $M_{\text {rad }}$ is approximately accounted for by the values of the coefficients.

II. The norms $\left|\boldsymbol{S}_{1}\right|,\left|\boldsymbol{S}_{2}\right|,|\tilde{\ell}|$ do not depend on the binary's separation $r$, with $\tilde{\ell}$ being defined as

$$
\tilde{\ell}(r) \equiv S_{\text {fin }}-\left[S_{1}(r)+S_{2}(r)\right]=L(r)-J_{\text {rad }}(r),
$$

where $S_{1}(r), S_{2}(r)$, and $\boldsymbol{L}(r)$ are the spins and the orbital angular momentum at separation $r$ and $J_{\text {rad }}(r)$ is the angular momentum radiated from $r$ to the merger. Clearly, $\boldsymbol{S}_{1}, \boldsymbol{S}_{2}$, and $\tilde{\boldsymbol{\ell}}$ can still depend on $r$ through their directions. While the constancy of $\left|\boldsymbol{S}_{1}\right|$ and $\left|\boldsymbol{S}_{2}\right|$ is a very good assumption for BHs, which do not have an internal structure, the constancy of $|\tilde{\ell}|$ is more heuristic and based on the idea that the merger takes place at an "effective" innermost stable circular orbit (ISCO), so that $|\tilde{\ell}|$ can be interpreted as the residual orbital angular momentum contributing to $S_{\text {fin }}$.

III. The final spin $\boldsymbol{S}_{\mathrm{fin}}$ is parallel to the initial total angular momentum $\boldsymbol{J}\left(r_{\text {in }}\right) \equiv \boldsymbol{S}_{1}\left(r_{\text {in }}\right)+\boldsymbol{S}_{2}\left(r_{\text {in }}\right)+\boldsymbol{L}\left(r_{\text {in }}\right)$. This amounts to assuming that $\boldsymbol{J}_{\mathrm{rad}}\left(r_{\text {in }}\right) \| \boldsymbol{J}\left(r_{\text {in }}\right)$. It replaces the assumption, made in Rezzolla et al. (2008a), that $\boldsymbol{J}_{\mathrm{rad}}\left(r_{\text {in }}\right) \| \boldsymbol{L}\left(r_{\text {in }}\right)$, which is only valid for a smaller set of configurations. We note that this assumption is motivated by PN theory: within the adiabatic approximation, the secular angular-momentum losses via gravitational radiation are along $\boldsymbol{J}$ (Apostolatos et al. 1994). This is because as $\boldsymbol{L}$ rotates around $\boldsymbol{J}$ the emission orthogonal to $\boldsymbol{J}$ averages out.

\footnotetext{
4 Recently, a new formula for the final spin has been proposed by Lousto et al. (2009). While that formula cannot be easily applied to generic configurations (because it depends on the final-plunge direction, which can only be determined via simulations), it reduces to a fit for aligned binaries. For the binaries considered above, it gives a much larger maximum error $\left|a_{\text {fin }}^{\mathrm{NR}}-a_{\text {fin }}^{\text {fit }}\right| \approx 0.05$.
}

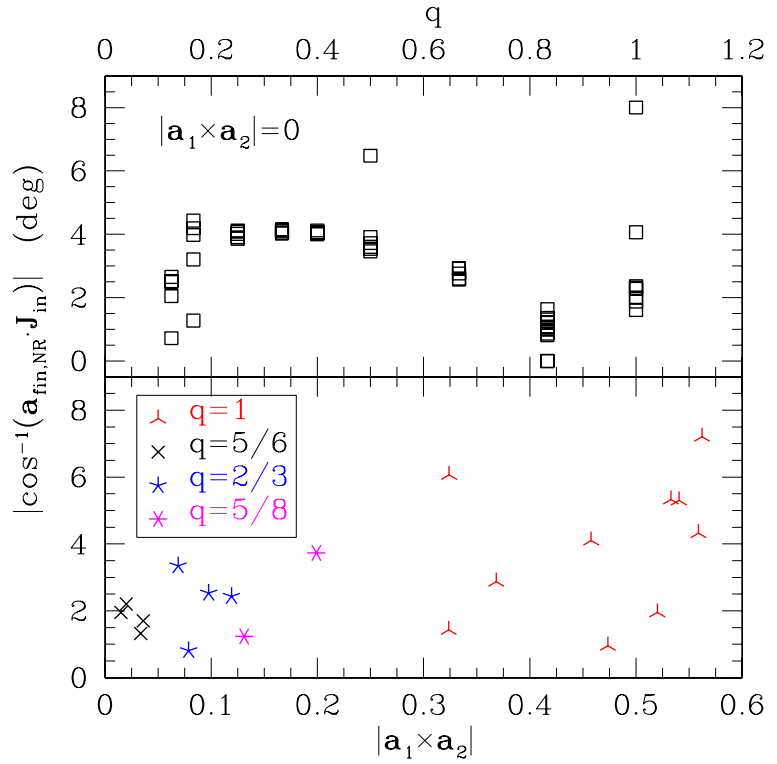

Figure 1. Accuracy of assumption (III) for all the generic binaries published so far, as a function of the spin-spin coupling "magnitude" $\left|\boldsymbol{a}_{1} \times \boldsymbol{a}_{2}\right|$ for the binaries with $\left|\boldsymbol{a}_{1} \times \boldsymbol{a}_{2}\right| \neq 0$ (lower panel), and of the mass ratio $q$ for the binaries with $\left|a_{1} \times a_{2}\right|=0$ (upper panel).

IV. The angle between $\boldsymbol{L}$ and $\boldsymbol{S} \equiv \boldsymbol{S}_{1}+\boldsymbol{S}_{2}$ and the angle between $\boldsymbol{S}_{1}$ and $\boldsymbol{S}_{1}$ are constant during the inspiral, although $\boldsymbol{L}$ and $\boldsymbol{S}$ precess around $\boldsymbol{J}$.

At 2.5 PN order, (III) and (IV) are approximately valid for any mass ratio if only one of the BHs is spinning, and for $M_{1}=M_{2}$ if one neglects spin-spin couplings. In both cases, in fact, $\boldsymbol{S}$ and $\boldsymbol{L}$ essentially precess around the direction $\hat{\boldsymbol{J}}$, which remains nearly constant (Apostolatos et al. 1994), and the angle between the two spins remains constant as well. The only case in which (III) and (IV) are not even approximately valid is for binaries which, at some point in the evolution, have $\boldsymbol{L}(r) \approx-\boldsymbol{S}(r)$. These orbits undergo the so-called "transitional precession" (Apostolatos et al. 1994), as a result of which $\hat{\boldsymbol{J}}$ changes significantly. Because transitional precession happens only if $\boldsymbol{L}$ and $\boldsymbol{S}$ are initially almost antialigned with $|\boldsymbol{L}|>|\boldsymbol{S}|$, it affects only a very small region of the parameter space, which is, moreover, poorly populated if the SMBHs are in a gas-rich environment (Bogdanovic et al. 2007).

Because assumption (III) plays a major role in our analysis, in Figure 1 we provide evidence of its (approximate) validity by using all the generic $\mathrm{BH}$-binary simulations (i.e., with spins not parallel to $\boldsymbol{L}$ ) published so far (Campanelli et al. 2007a, 2007b; Lousto \& Zlochower 2009; Tichy \& Marronetti 2008), except two binaries in Campanelli et al. (2009), which, when reproduced by us, seem affected by imprecisions (M. Jasiulek 2009, private communication). In particular, we plot the angle between $\boldsymbol{S}_{\text {fin }}$ and $\boldsymbol{J}\left(r_{\text {in }}\right)$ as a function of the spin-spin coupling "magnitude" $\left|\boldsymbol{a}_{1} \times \boldsymbol{a}_{2}\right|$ for the binaries with $\left|\boldsymbol{a}_{1} \times \boldsymbol{a}_{2}\right| \neq 0$ (lower panel), and of $q$ for the binaries with $\left|\boldsymbol{a}_{1} \times \boldsymbol{a}_{2}\right|=0$ (upper panel). Clearly, assumption (III) is valid to within 4-5 degrees, with maximum errors of 7-8 degrees. These errors are compatible with the accuracy with which the NR simulations can compute the final-spin direction for strongly precessing binaries (note the error's noisy dependence on $\left.\left|\boldsymbol{a}_{1} \times \boldsymbol{a}_{2}\right|\right)$.

$\mathrm{V}$. When the initial spin vectors are equal and opposite and the masses are equal, the spin of the final BH is the same as for non-spinning binaries. Besides being physically reasonablereflecting the expectation that if the spins are equal and 
opposite their contributions cancel out-this assumption is confirmed by NR simulations (see discussion in Rezzolla 2009) and by the leading-order PN spin-spin and spin-orbit couplings.

With these assumptions, we derive an expression for the final spin. Let us write Equation (4), using (I), as

$$
\boldsymbol{a}_{\mathrm{fin}}=\frac{1}{(1+q)^{2}}\left(\boldsymbol{a}_{1}(r)+\boldsymbol{a}_{2}(r) q^{2}+\boldsymbol{\ell}(r) q\right),
$$

where $\boldsymbol{a}_{\mathrm{fin}}=\boldsymbol{S}_{\mathrm{fin}} / M^{2}$ and $\boldsymbol{\ell} \equiv \tilde{\boldsymbol{\ell}} /\left(M_{1} M_{2}\right)$. Using (II), the finalspin norm is

$$
\begin{aligned}
& \left|\boldsymbol{a}_{\text {fin }}\right|=\frac{1}{(1+q)^{2}}\left[\left|\boldsymbol{a}_{1}\right|^{2}+\left|\boldsymbol{a}_{2}\right|^{2} q^{4}+2\left|\boldsymbol{a}_{2}\right|\left|\boldsymbol{a}_{1}\right| q^{2} \cos \alpha\right. \\
& \left.+2\left(\left|\boldsymbol{a}_{1}\right| \cos \beta(r)+\left|\boldsymbol{a}_{2}\right| q^{2} \cos \gamma(r)\right)|\boldsymbol{\ell}| q+|\boldsymbol{\ell}|^{2} q^{2}\right]^{1 / 2}
\end{aligned}
$$

where $\alpha, \beta$, and $\gamma$ are

$$
\cos \alpha \equiv \hat{\boldsymbol{a}}_{1} \cdot \hat{\boldsymbol{a}}_{2}, \cos \beta \equiv \hat{\boldsymbol{a}}_{1} \cdot \hat{\boldsymbol{\ell}}, \cos \gamma \equiv \hat{\boldsymbol{a}}_{2} \cdot \hat{\boldsymbol{\ell}}
$$

Note that because of (IV), $\alpha$ does not depend on the separation and is simply the angle between the spins at the initial separation $r_{\text {in }}$. The angles $\beta$ and $\gamma$ are instead functions of the binary's separation, but this dependence cancels out in the linear combination in which they appear in Equation (6), which is indeed, within the assumptions made, independent of the separation and which can therefore be evaluated at $r=r_{\text {in }}$. To see this, let us consider Equation (6) at the effective ISCO. There, $\boldsymbol{J}_{\text {rad }}\left(r_{\text {ISCO }}\right)=0$ by definition and therefore $\boldsymbol{\ell}\left(r_{\text {ISCO }}\right)=\boldsymbol{L}\left(r_{\text {ISCO }}\right)$. As a result, $\beta\left(r_{\text {ISCO }}\right)$ $\left[\gamma\left(r_{\text {ISCO }}\right)\right]$ are simply the angles between $S_{1}\left[S_{2}\right]$, and $\boldsymbol{L}$ at the ISCO. Using now assumption (IV), we can write part of Equation (6) as

$$
\begin{gathered}
\left|\boldsymbol{a}_{1}\right| \cos \beta\left(r_{\mathrm{ISCO}}\right)+\left|\boldsymbol{a}_{2}\right| q^{2} \cos \gamma\left(r_{\mathrm{ISCO}}\right)=(\hat{\boldsymbol{L}} \cdot \boldsymbol{S})_{\mathrm{ISCO}} / M_{1}^{2} \\
=(\hat{\boldsymbol{L}} \cdot \boldsymbol{S}) / M_{1}^{2}=\left|\boldsymbol{a}_{1}\right| \cos \tilde{\beta}(r)+\left|\boldsymbol{a}_{2}\right| q^{2} \cos \tilde{\gamma}(r),
\end{gathered}
$$

where $\widetilde{\beta}$ and $\tilde{\gamma}$ are the angles between the spins and $\boldsymbol{L}$ at any separation $r$ and thus also at $r=r_{\text {in }}$

$$
\cos \widetilde{\beta} \equiv \hat{\boldsymbol{a}}_{1} \cdot \hat{\boldsymbol{L}}, \quad \cos \widetilde{\gamma} \equiv \hat{\boldsymbol{a}}_{2} \cdot \hat{\boldsymbol{L}}
$$

This proves our previous statement: the dependence on $r$ that $\beta$ and $\gamma$ have in Equation (6) is canceled by the linear combination in which they appear. Stated differently, the finalspin norm is simply given by Equation (6) where $\beta(r) \rightarrow \widetilde{\beta}\left(r_{\text {in }}\right)$ and $\gamma(r) \rightarrow \tilde{\gamma}\left(r_{\text {in }}\right)$. Thus, one does not need to worry about the angles between $\hat{\boldsymbol{a}}_{1,2}$ and $\hat{\boldsymbol{\ell}}$ but simply about the angles between $\hat{\boldsymbol{a}}_{1,2}$ and $\hat{\boldsymbol{L}}$ at $r=r_{\text {in }}$, which are easy to compute.

Finally, we need to compute $|\ell|$ and for this we proceed like in Rezzolla et al. (2008a) and match Equation (6) at $r=r_{\text {ISCo }}$ with Equation (1) for parallel and aligned spins $\left(\alpha=\beta\left(r_{\text {ISCO }}\right)=\gamma\left(r_{\text {ISCO }}\right)=0\right)$, for parallel and antialigned spins $\left(\alpha=0, \beta\left(r_{\text {ISCO }}\right)=\gamma\left(r_{\text {ISCO }}\right)=\pi\right)$, and for antiparallel spins which are aligned or antialigned $\left(\alpha=\beta\left(r_{\text {ISCO }}\right)=\pi, \gamma\left(r_{\text {ISCO }}\right)=0\right.$ or $\left.\alpha=\gamma\left(r_{\text {ISCO }}\right)=\pi, \beta\left(r_{\text {ISCO }}\right)=0\right)$. As noted in Rezzolla et al. (2008a), this matching is not unique, but the degeneracy can be broken by exploiting assumption (V) (i.e., by imposing that $|\boldsymbol{\ell}|$ does not depend on $\boldsymbol{a}_{1,2}$ when $\boldsymbol{a}_{1}=-\boldsymbol{a}_{2}$ and $\left.q=1\right)$ and by requiring for simplicity that $|\boldsymbol{\ell}|$ depends linearly on $\cos \alpha$, $\cos \beta$, and $\cos \gamma$. Using these constraints and Equation (8), we obtain again an expression valid for any separation and hence for $r=r_{\text {in }}$

$$
\begin{aligned}
|\ell|= & 2 \sqrt{3}+t_{2} v+t_{3} v^{2}+\frac{s_{4}}{\left(1+q^{2}\right)^{2}} \\
& \left.\times\left(\left|\boldsymbol{a}_{1}\right|^{2}+\left|\boldsymbol{a}_{2}\right|^{2} q^{4}+2\left|\boldsymbol{a}_{1}\right|\left|\boldsymbol{a}_{2}\right| q^{2} \cos \alpha\right)\right)+\left(\frac{s_{5} v+t_{0}+2}{1+q^{2}}\right) \\
& \times\left(\left|\boldsymbol{a}_{1}\right| \cos \tilde{\beta}\left(r_{\text {in }}\right)+\left|\boldsymbol{a}_{2}\right| q^{2} \cos \tilde{\gamma}\left(r_{\text {in }}\right)\right) .
\end{aligned}
$$

When comparing Equations (6), (8), and (10) with Equations (8) and (11) of Rezzolla et al. (2008a), it is straightforward to realize that they are mathematically the same, although derived under a different (and improved) set of assumptions. This is simply because the new assumptions (II) and (IV) are compatible, via Equation (8), with the old ones. However, the new assumptions make a substantial difference in the prediction of the final-spin direction. Since (III) states that $\boldsymbol{a}_{\text {fin }} \| \boldsymbol{J}\left(r_{\text {in }}\right)$, the angle $\theta_{\text {fin }}$ between the final spin and the initial orbital angular momentum $\boldsymbol{L}\left(r_{\text {in }}\right)$ is given by

$$
\cos \theta_{\text {fin }}=\hat{\boldsymbol{L}}\left(r_{\text {in }}\right) \cdot \hat{\boldsymbol{J}}\left(r_{\text {in }}\right) .
$$

This expression replaces and improves Equation (10) of Rezzolla et al. (2008a) and, as we will show, is verified both for initial separations of a few gravitational radii, such as those considered in NR, and for larger separations (e.g., $\sim 10^{4} M$ ), which are relevant for cosmological applications.

\section{COMPARISON WITH NUMERICAL-RELATIVITY DATA}

We now test our Equations (6), (8), and (10) for $\left|\boldsymbol{a}_{\text {fin }}\right|$ and our Equation (11) for $\theta_{\text {fin }}$ against the NR simulations published so far for generic binaries. Note that to test the expression for $\theta_{\text {fin }}$ we use the same simulations as in Figure 1 (i.e., Campanelli et al. 2007a, 2007b; Lousto \& Zlochower 2009; Tichy \& Marronetti 2008), while to test the expression for $\left|\boldsymbol{a}_{\text {fin }}\right|$ we also use the data of Tichy \& Marronetti (2007) (which only reports the final-spin norm). Also, we compare our predictions (AEI) with those of similar formulas suggested by Buonanno et al. (2008) (BKL), Rezzolla et al. (2008a) ( $\left.\mathrm{AEI}_{\text {old }}\right)$, and Tichy \& Marronetti (2008) (FAU). The comparison consists of two steps. First, we use as input the initial data of the NR simulations, in which the binaries have small separations (i.e., $r_{\text {in }} \lesssim 10 M$ ). Second, we use binaries at large separations (i.e., $r_{\text {in }} \leqslant 2 \times 10^{4} M$ ), for which the dynamics starts being dominated by gravitationalwave emission and which are therefore of direct relevance for cosmological investigations. More precisely, we evolve the NR initial configurations back in time up to a separation of $2 \times 10^{4} \mathrm{M}$ using the $2.5 \mathrm{PN}$ equations in the quasi-circular limit (Buonanno et al. 2003), calculating the predictions of the different formulas at each step and considering the maximum error for each formula. (We stress, however, that the figures we present do not change significantly if we integrate only up to a separation of $\sim 200 \mathrm{M}$.)

The left panel of Figure 2 shows the predictions of the various formulas for $\left|\boldsymbol{a}_{\text {fin }}\right|$, when applied to the small-separation configurations corresponding to the initial data of the NR simulations. In particular, it reports the error in the final-spin norm || $\boldsymbol{a}_{\mathrm{fin}, \mathrm{NR}}|-| \boldsymbol{a}_{\mathrm{fin}, *}||-$ where "**" stands for "AEI" (which, as already stressed, gives the same predictions for $\left|\boldsymbol{a}_{\mathrm{fin}}\right|$ as "AEI ${ }_{\text {old }}$ "), "FAU," or "BKL" — as a function of $q$. The right panel shows instead the maximum error when the configurations are 

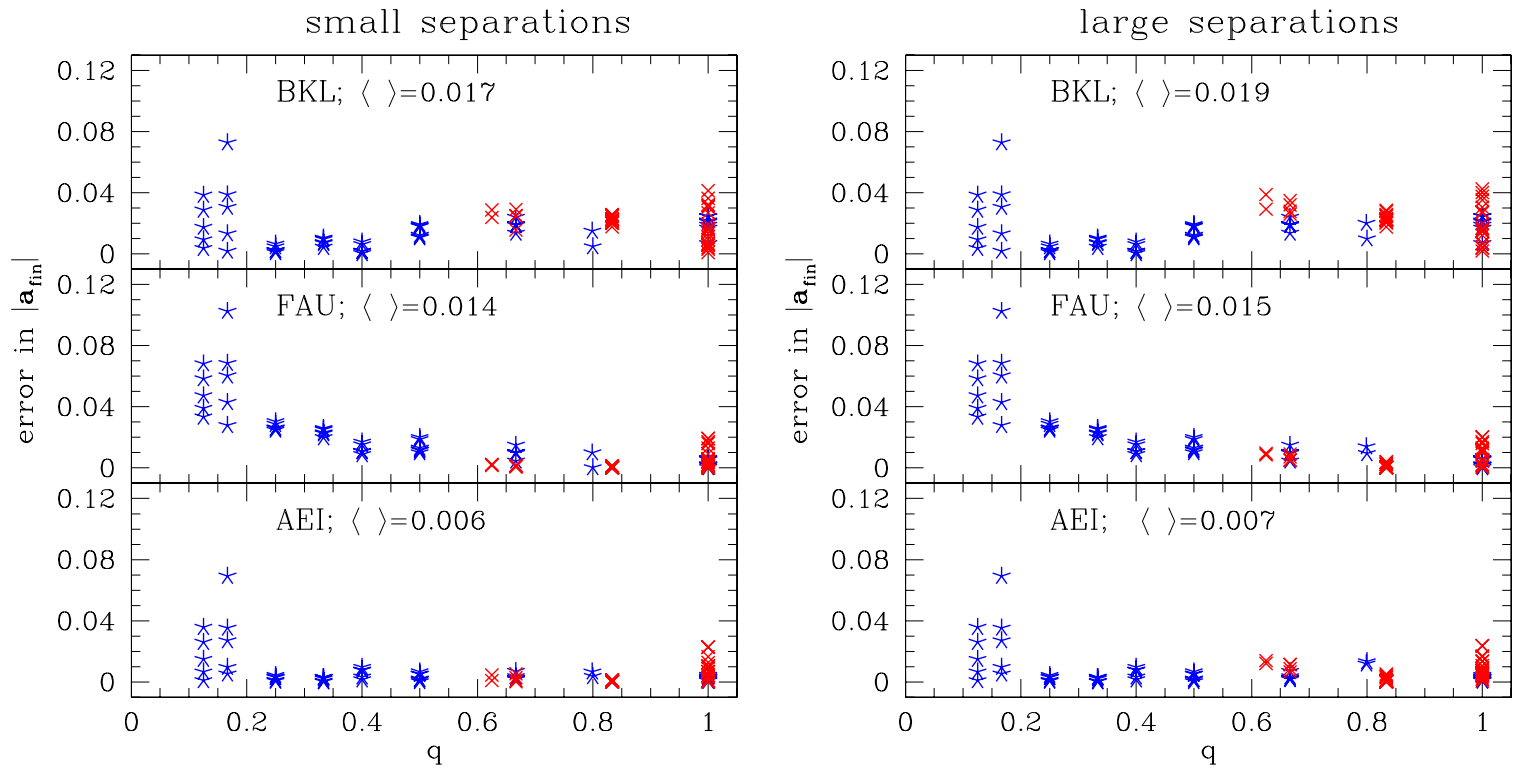

Figure 2. Left panel: error in the final-spin norm || $\boldsymbol{a}_{\mathrm{fin}, \mathrm{NR}}|-| \boldsymbol{a}_{\mathrm{fin}, *} \|$ ("**" being either "AEI," "FAU," or "BKL") as a function of the mass ratio, when the various formulas are applied to the small-separation configurations corresponding to the initial data of the 45 simulations of Lousto \& Zlochower (2009), Campanelli et al. (2007a, 2007b) (blue stars), and to the 41 simulations of Tichy \& Marronetti (2008, 2007) (red crosses). The numbers next to each label indicate the average error. Right panel: the same as in the left one but for binaries at large separations of $r=2 \times 10^{4} M$. Although the new AEI expression is slightly better, all formulas give accurate predictions for $\left|\boldsymbol{a}_{\mathrm{fin}}\right|$ both for small and large separations. The larger errors for small $q$ are most likely due to the larger truncation errors affecting those simulations (Lousto \& Zlochower 2009).
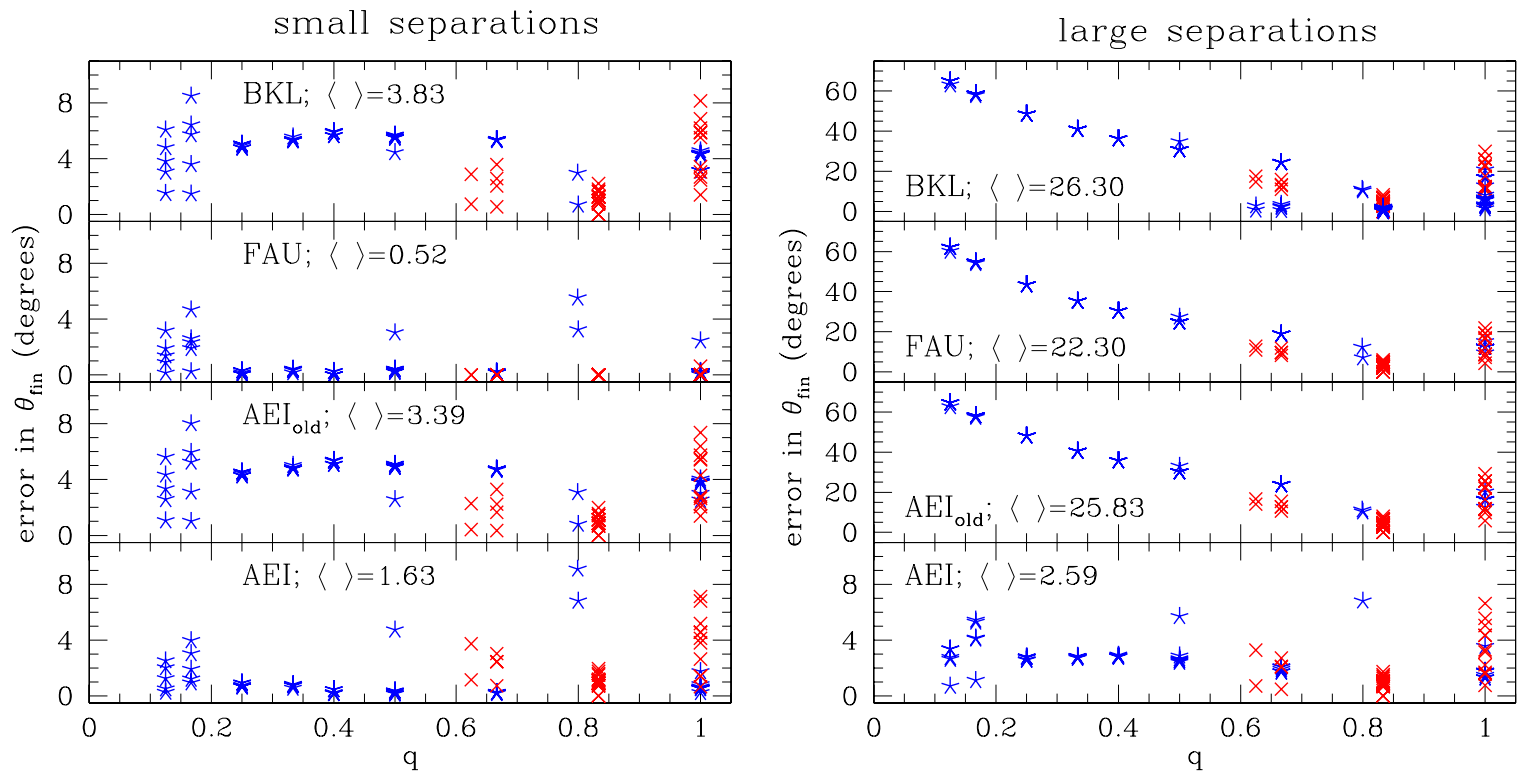

Figure 3. Same as in Figure 2, but for the inclination-angle error $\left|\theta_{\mathrm{fin}, *}-\arccos \left[\hat{\boldsymbol{L}}\left(r_{\mathrm{in}}\right) \cdot \hat{\boldsymbol{a}}_{\mathrm{fin}, \mathrm{NR}}\right]\right|$ and without the eight binaries of Tichy \& Marronetti (2007), for which the final-spin direction was not published. Here too, the larger errors for small-separation binaries with small $q$ are likely due to the larger truncation errors. The new AEI expression is accurate both for small and large separations, while the other ones become imprecise for large separations.

evolved back in time up to $r_{\text {in }}=2 \times 10^{4} M$. Figure 2 clearly shows that although the AEI expression is slightly better, one can apply the old formulas to widely separated binaries if the focus is on $\left|\boldsymbol{a}_{\text {fin }}\right|$ only. Note that the larger errors for small $q$ are most likely due to the larger truncation errors affecting those simulations (see Lousto \& Zlochower 2009, Section IIIA).

However, the situation is very different for the final-spin direction. In particular, Figure 3 reports the inclination-angle error $\left|\theta_{\mathrm{fin}, *}-\arccos \left[\hat{\boldsymbol{L}}\left(r_{\mathrm{in}}\right) \cdot \hat{\boldsymbol{a}}_{\mathrm{fin}, \mathrm{NR}}\right]\right|$, for all the data in Figure 2, except those of Tichy \& Marronetti (2007), for which the finalspin direction was not published. When considering smallseparation binaries (left panel), our new formula performs slightly better than the "BKL" and "AEI old " formulas, but it is not better than the "FAU" one. Indeed, the latter is exact by construction for the 32 binaries of Table II in Tichy \& Marronetti (2008), because for such data the final-spin direction was not published and has been here reconstructed using the FAU formula. When applied to large-separation binaries (right panel), however, our new formula performs much better than the other ones. This result is not surprising since all the previous formulas predict the same value of $\theta_{\text {fin }}$ for all $r_{\text {in }}$, as they neglect the precession of the binary's orbital plane. As suggested by Tichy \& Marronetti (2007), one can in principle use PN theory to integrate the binary to small separations, and then apply the old 
formulas. As already mentioned, however, this is impractical, compared to our simple and algebraic expressions for the final spin. Also, note that the error in $\theta_{\text {fin }}$ made by the previous formulas at large separations increases for small- $q$ binaries. This is again because the previous formulas predict the same $\theta_{\text {fin }}$ for all $r_{\text {in }}$. Therefore, because the correct $\theta_{\text {fin }}$ becomes small for large $r_{\text {in }}\left(\right.$ since $\left.\cos \left(\theta_{\text {fin, NR }}\right) \approx \hat{\boldsymbol{L}}\left(r_{\text {in }}\right) \cdot \hat{\boldsymbol{J}}\left(r_{\text {in }}\right) \approx 1\right)$, the maximum error of the previous formulas is roughly given by their prediction for $\theta_{\text {fin }}$ at small separations, which can be large for small $q$ if the angle between $\hat{\boldsymbol{S}}$ and $\hat{\boldsymbol{L}}$ at small separations is large (because for $q \approx 0, \hat{\boldsymbol{J}}\left(r_{\text {in }}\right) \approx \hat{\boldsymbol{S}}\left(r_{\text {in }}\right)$ and $\left.\cos \left(\theta_{\text {fin }}\right) \approx \hat{\boldsymbol{L}}\left(r_{\text {in }}\right) \cdot \hat{\boldsymbol{S}}\left(r_{\text {in }}\right)\right)$.

The very small errors in the predictions of the final-spin direction also provide additional evidence, besides that in Figure 1, of the validity of assumption (III), namely that $\hat{\boldsymbol{S}}_{\mathrm{fin}} \simeq \hat{\boldsymbol{J}}\left(r_{\mathrm{in}}\right)$. Also, they suggest a correlation between the finalspin direction and the orbital plane when the binary was widely separated. Stated differently, by observing $\hat{\boldsymbol{a}}_{\text {fin }}$, e.g., via a jet if this is assumed along $\hat{\boldsymbol{a}}_{\text {fin }}$, one is virtually "observing" $\hat{\boldsymbol{J}}\left(r_{\text {in }}\right)$ and can conclude that the orbital plane at large separations was roughly orthogonal to the final spin. Our result could therefore be applied to X-shaped radio sources, for which the origin of the double pair of jets is under debate (Capetti et al. 2002; Merritt \& Ekers 2002).

\section{CONCLUSIONS}

We have derived a new formula predicting the spin of the $\mathrm{BH}$ resulting from the merger of two BHs in quasi-circular orbits and having arbitrary initial masses and spins. Our derivation is based on a revised set of assumptions and exploits an additional constraint to reduce to only four the number of undetermined coefficients. The new formula is identical to that proposed in Rezzolla et al. (2008a) in the prediction of the final-spin norm, but is different in the prediction of its direction, showing a much better agreement with the numerical data. The new formula can be applied to binaries with separations larger than $\sim 200 \mathrm{M}$ without any preliminary integration of the PN equations, in contrast with what would be needed by the other formulas proposed in the literature. Thus, our formula is particularly suitable for astrophysical and cosmological applications and could provide clues about the relation between the spin of the $\mathrm{SMBH}$ in the center of active galactic nuclei and the binary's orbital plane well before the merger.
We are grateful to M. Jasiulek, L. A. Gergely, and A. Buonanno for helpful discussions. E.B. acknowledges support from NSF grant PHY-0603762.

\section{REFERENCES}

Apostolatos, T. A., Cutler, C., Sussman, G. J., \& Thorne, K. S. 1994, Phys. Rev. D, 49, 6274

Berti, E., Cardoso, J., Cardoso, V., \& Cavaglià, M. 2007a, Phys. Rev. D, 76, 104044

Berti, E., Cardoso, V., González, J. A., Sperhake, U., \& Brügmann, B. 2008, Class. Quantum Grav., 25, 114035

Berti, E., Cardoso, V., Gonzalez, J. A., Sperhake, U., Hannam, M., Husa, S., \& Brügmann, B. 2007b, Phys. Rev. D, 76, 064034

Berti, E., \& Volonteri, M. 2008, ApJ, 684, 822

Bogdanovic, T., Reynolds, C. S., \& Miller, M. C. 2007, ApJ, 661, L147

Boyle, L., \& Kesden, M. 2008, Phys. Rev. D, 78, 024017

Boyle, L., Kesden, M., \& Nissanke, S. 2008, Phys. Rev. Lett., 100, 151101

Buonanno, A., Chen, Y., \& Vallisneri, M. 2003, Phys. Rev. D, 67, 104025

Buonanno, A., Kidder, L. E., \& Lehner, L. 2008, Phys. Rev. D, 77, 026004

Buonanno, A., Pan, Y., Baker, J. G., Centrella, J., Kelly, B. J., McWilliams, S. T., \& van Meter, J. R. 2007, Phys. Rev. D, 76, 104049

Buonanno, A., Pan, Y., Pfeiffer, H. P., Scheel, M. A., Buchman, L. T., \& Kidder, L. E. 2009, Phys. Rev. D, 79, 124028

Campanelli, M., Lousto, C. O., Nakano, H., \& Zlochower, Y. 2009, Phys. Rev. D, 79, 084010

Campanelli, M., Lousto, C. O., Zlochower, Y., Krishnan, B., \& Merritt, D. 2007a, Phys. Rev. D, 75, 064030

Campanelli, M., Lousto, C., Zlochower, Y., \& Merritt, D. 2007b, ApJ, 659, L5

Capetti, A., Zamfir, S., Rossi, P., Bodo, G., Zanni, C., \& Massaglia, S. 2002, A\&A, 394, 39

Damour, T., \& Nagar, A. 2009, arXiv:0902.0136

Gonzalez, J. A., Sperhake, U., \& Bruegmann, B. 2009, Phys. Rev. D, 79, 124006

Hughes, S. A., \& Blandford, R. D. 2003, ApJ, 585, L101

Kesden, M. 2008, Phys. Rev. D, 78, 084030

Lousto, C. O., Campanelli, M., \& Zlochower, Y. 2009, arXiv:0904.3541

Lousto, C. O., \& Zlochower, Y. 2009, Phys. Rev. D, 79, 064018

Merritt, D., \& Ekers, R. D. 2002, Science, 297, 1310

Miller, J. M., Reynolds, C. S., Fabian, A. C., Miniutti, G., \& Gallo, L. C. 2009, arXiv:0902.2840

Pretorius, F. 2007, Relativistic Objects in Compact Binaries: From Birth to Coalescence (Berlin: Springer Verlag)

Rezzolla, L. 2009, Class. Quantum Grav., 26, 094023

Rezzolla, L., Barausse, E., Dorband, E. N., Pollney, D., Reisswig, C., Seiler, J., \& Husa, S. 2008a, Phys. Rev. D, 78, 044002

Rezzolla, L., Diener, P., Dorband, E. N., Pollney, D., Reisswig, C., Schnetter, E., \& Seiler, J. 2008b, ApJ, 674, L29

Rezzolla, L., Dorband, E. N., Reisswig, C., Diener, P., Pollney, D., Schnetter E., \& Szilágyi, B. 2008c, ApJ, 679, 1422

Scheel, M. A., Boyle, M., Chu, T., Kidder, L. E., Matthews, K. D., \& Pfeiffer, H. P. 2009, Phys. Rev. D, 79, 024003

Tichy, W., \& Marronetti, P. 2007, Phys. Rev. D, 76, 061502

Tichy, W., \& Marronetti, P. 2008, Phys. Rev. D, 78, 081501 